18 Shen H, Tang Y, Huang L, Yang R, Wu Y, Wang P et al. Applications of diffusionweighted MRI in thoracic spinal cord injury without radiographic abnormality. Int Orthop 2007; 31: 375-383.

19 Xing W, Wang X, Han Z, Liao W, Liu F. Diffusion-weighted magnetic resonance imaging for acute trauma of the spine cord. Zhong Nan Da Xue Xue Bao Yi Xue Ban 2010; 35: 760-765.

20 Tsuchiya K, Fujikawa A, Honya K, Tateishi H, Nitatori T. Value of diffusion-weighted MR imaging in acute cervical cord injury as a predictor of outcome. Neuroradiology 2006; 48: 803-808.

21 Sagiuchi T, Tachibana S, Endo M, Hayakawa K. Diffusion-weighted MRI of the cervical cord in acute spinal cord injury with type II odontoid fracture. J Comput Assist Tomogr 2002; 26: 654-656

22 Cihangiroglu M, Citci B, Kilickesmez O, Firat Z, Karlỳkaya G, Ulua AM et al. The utility of high b-value DWI in evaluation of ischemic stroke at 3T. Eur J Radiol 2011; 78: 75-81.

23 Shepard MJ, Bracken MB. Magnetic resonance imaging and neurological recovery in acute spinal cord injury: observations from the National Acute Spinal Cord Injury Study 3. Spinal Cord 1999; 37: 833-837.

24 Catz A, Itzkovich M, Agranov E, Ring H, Tamir A. The spinal cord independence measure (SCIM): sensitivity to functional changes in subgroups of spinal cord lesion patients. Spinal Cord 2001; 39: 97-100.

25 Marcel C, Kremer S, Jeantroux J, Blanc F, Dietemann JL, De Sèze J. Diffusion-weighted imaging in noncompressive myelopathies: a 33-patient prospective study. J Neurol 2010; 257: 1438-1445.
26 Kremer S, Oppenheim C, Schmitt E, Dietemann JL. Diffusion MRI: technique and clinical applications. J Radiol 2007; 88: 428-443.

27 Rowland JW, Hawryluk GW, Kwon B, Fehlings MG. Current status of acute spinal cord injury pathophysiology and emerging therapies: promise on the horizon. Neurosurg Focus 2008; 25: E2.

28 Saadoun S, Papadopoulos MC. Aquaporin-4 in brain and spinal cord oedema. Neuroscience 2010; 168: 1036-1046.

29 Bammer R, Augustin M, Prokesch RW, Stollberger R, Fazekas F. Diffusion-weighted imaging of the spinal cord: interleaved echo-planar imaging is superior to fast spinecho. J Magn Reson Imaging 2002; 15: 364-373.

30 Mamata H, Jolesz FA, Maier SE. Apparent diffusion coefficient and fractional anisotropy in spinal cord: age and cervical spondylosis-related changes. J Magn Reson Imaging 2005; 22: 38-43.

31 Schwartz ED, Cooper ET, Chin CL, Wehrli S, Tessler A, Hackney DB. Ex vivo evaluation of ADC values within spinal cord white matter tracts. AJNR Am J Neuroradiol 2005; 26: 390-397.

32 Schwartz ED, Chin CL, Shumsky JS, Jawada AF, Kooper Brownb B, Wehrlic S et al. Apparent diffusion coefficients in spinal cord transplants and surrounding white matter correlate with degree of axonal dieback after injury in rats. AJNR Am J Neuroradiol 2005; 26: 7-18.

33 Quencer RM, Pattany PM. Diffusion-weighted imaging of the spinal cord: is there a future? AJNR Am J Neuroradiol 2000; 21: 1181-1182.

34 Thurnher MM, Law M. Diffusion-weighted imaging, diffusion-tensor imaging, and fiber tractography of the spinal cord. Magn Reson Imaging Clin N Am 2009; 17: 225-244.

\title{
Editorial Note on: Diagnostic value of diffusion-weighted MR imaging for acute stage of spine cord injury
}

Spinal Cord (2012) 50, 431; doi:10.1038/sc.2011.172; published online 7 February 2012

Editorial Note on: Spinal Cord (2012) 50, 426-431; doi:10.1038/ sc.2011.168; published online 7 February 2012

This paper is about the application of conventional magnetic resonance imaging (MRI) and diffusion-weighted imaging (DWI) on traumatic spinal cord injury (tSCI) within $24 \mathrm{~h}$. It is really a challenge to perform DWI in the spinal cord. Therefore, only a few papers reported DWI in the early stage of tSCI. With the limited samples, this study claimed that 'T2-weighted and DW imaging have comparable detection rates for spinal cord damage'.

DWI offers a promising approach to detect axon integrity in spinal cord based on the water mobility along the waterproof myelin structure, mainly beneficial for the evaluation of white matter integrity. It might not be appropriate to apply DWI to detect the changes in gray matter. It could be one of the possible reasons why DWI did not show difference in the present article, as the hematoma in gray matter is a major feature within the first $24 \mathrm{~h}$.

However, several previous studies proved that DWI is indeed a powerful tool with high sensitivity than conventional MRI. Diffusion MRI should provide higher sensitivity and diagnostic value in the early stage of tSCI. The present study excluded a paper (reference 18) that is about the application of DWI on tSCI within $48 \mathrm{~h}$. It is important to note that DWI can detect the abnormality in the spinal cord much earlier than conventional MRI. It is worth applying DWI to accurately predict the initial damage of the spinal cord.

Apart from the known technical challenges, including the susceptibility to motion artifacts and small size of spinal cord, the varied protocols for DWI adopted in different studies makes it difficult to conclude a consistent result. With the technical development, the diffusion tensor imaging has gained great merits in both research and clinical field with widespread availability. Therefore, as the authors cited in the limitation, the use of advanced acquisition techniques is highly appreciated in the future study.

\section{CONFLICT OF INTEREST}

The author declares no conflict of interest.

$\mathrm{Y} \mathrm{Hu}$

Department of Orthopaedics and Traumatology, LKS Faculty of Medicine, The University of Hong Kong,

Hong Kong, China

E-mail:yhud@hkusua.hku.hk 